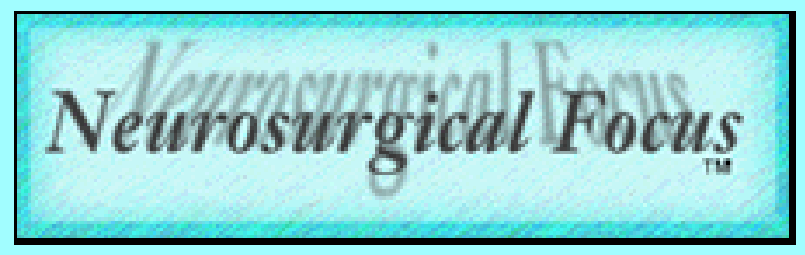

\title{
Commentary by Anthony J. Caputy, M.D.
}

The indications for spinal fusion and instrumentation are relatively similar in all four manuscripts. The percentage of patients meeting this criteria for fusion is different. This may be explained by the differences in the patient population in the four reviews. We limited our population to elderly patients with degenerative stenosis. The other manuscripts have incorporated patients with isthmic spondylolisthesis, instability, secondary trauma, neoplasm, and infection. We have found that the over all fusion rate for patients undergoing decompression for neurological symptoms at unstable segments should be no greater than $15 \%$.

Recently, there has been an explosion of interbody cages created to augment posterior lumbar interbody fusion in patients with lumbar stenosis. The role of these devices in patients with lumbar stenosis has not been proven, and their use must be carefully evaluated. 